\title{
Evaluation of a compact sensor for backscattering and absorption
}

\author{
Alina Gainusa Bogdan* and Emmanuel S. Boss \\ University of Maine, 458 Aubert Hall, Orono, Maine 04469, USA \\ *Corresponding author: alina.gainusabogdan@maine.edu
}

Received 7 December 2010; revised 20 March 2011; accepted 24 April 2011; posted 18 May 2011 (Doc. ID 139208); published 11 July 2011

\begin{abstract}
Seawater inherent optical properties (IOPs) are key parameters in a wide range of applications in environmental studies and oceanographic research. In particular, the absorption coefficient $(a)$ is the typical IOP used to obtain the concentration of chlorophyll- $a$ in the water-a critical parameter in biological oceanography studies and the backscattering coefficient $\left(b_{b}\right)$ is used as a measure of turbidity. In this study, we test a novel instrument concept designed to obtain both the absorption and backscattering coefficients. The instrument would emit a collimated monochromatic light beam into the water retrieving the backscattered light intensity as a function of distance from the center of illumination. We use Monte Carlo modeling of light propagation to create an inversion algorithm that translates the signal from such an instrument into values of $a$ and $b_{b}$. Our results, based on simulations spanning the bulk of natural values of seawater IOP combinations, indicate that a $6.2 \mathrm{~cm}$ diameter instrument with a radial resolution of $1 \mathrm{~cm}$ would be capable of predicting $b_{b}$ within less than $13.4 \%$ relative difference and $a$ within less than $57 \%$ relative difference (for $90 \%$ of the inverted $a$ values, the relative errors fall below $29.7 \%$ ). Additionally, these errors could be further reduced by constraining the inversion algorithm with information from concurrent measurements of other IOPs. Such a compact and relatively simple device could have multiple applications for in situ optical measurements, including $a$ and $b_{b}$ retrievals from instrumentation mounted on autonomous underwater vehicles. Furthermore, the same methodology could possibly be used for an out-of-water sensor. (C) 2011 Optical Society of America

OCIS codes: $\quad 010.0280,010.1030,010.1350,010.4450,010.5620$.
\end{abstract}

\section{Introduction}

Inherent optical properties (IOPs) of aquatic environments fully determine the way light propagates through water. By definition, independent of the ambient light field, aquatic IOPs carry the signatures of each component of the water mass (the water molecules themselves, the suspended particles, and the dissolved constituents) and as such can be used to retrieve information about these components. Such information has applications in several different fields, such as water quality assessment (e.g., for drinking water or oyster farming [1]), ecology and human health (e.g., detection of harmful algal blooms $[2, \underline{3}]$ ), and oceanography (e.g., characterization of

0003-6935/11/213758-15\$15.00/0

(C) 2011 Optical Society of America biogeochemical processes in aquatic environments, underwater visibility studies, calibration and interpretation of satellite ocean color [2-4]).

Some seawater IOPs can be estimated from optical information retrieved from space by satellite-based sensors. While providing extremely valuable spatial coverage, these measurements have relatively limited spatial and temporal resolution, can only provide surface information, and currently still need in situ validation. Many current in situ IOP sensors are still rather cumbersome due to their size. Additionally, some require a flow-through system, which introduces more complexity to the measurements (and thus, sources of uncertainty), a higher power demand, and higher maintenance needs.

Ideal in situ instruments to measure these properties should be small (for easy deployment on different platforms), have low power demands, and minimize 
fouling (for long-term measurements). Here, we test a novel instrument concept inspired by the Thor instrument used in atmospheric studies [5] that should be capable of simultaneously measuring two key IOPs: the absorption coefficient ( $a$, used, for example, to retrieve chlorophyll- $a$ concentrations in the water for biological oceanography applications [6]) and the backscattering coefficient $\left(b_{b}\right.$, used to measure turbidity, a critical parameter in water quality assessment [7]).

The concept can be illustrated by a simple laboratory experiment (see Fig. 1). Directing a laser beam straight onto the flat surfaces of solutions with different absorption and scattering coefficients results in backscattering "spots" of different sizes and intensities (observer located next to the light source). Increasing the scattering coefficient increases scattering both in the backward and sideway directions relative to the incident beam. Therefore, theoretically, at a higher scattering coefficient, both the total intensity of backscattered light and its lateral diffusion should increase. Increasing the absorption coefficient reduces intensity with the traversed path length. This should result in both a decreased total intensity of backscattered light and a narrower lateral distribution, as light scattered first sideways and then back toward the observer has an increased path length with greater distance traveled from the incident beam (and hence a larger probability to be absorbed).

Experimentally (Fig. 1), increasing the absorption coefficient from $a_{0}$ through $a_{2}$ decreases the total backscattered light intensity. Increasing the

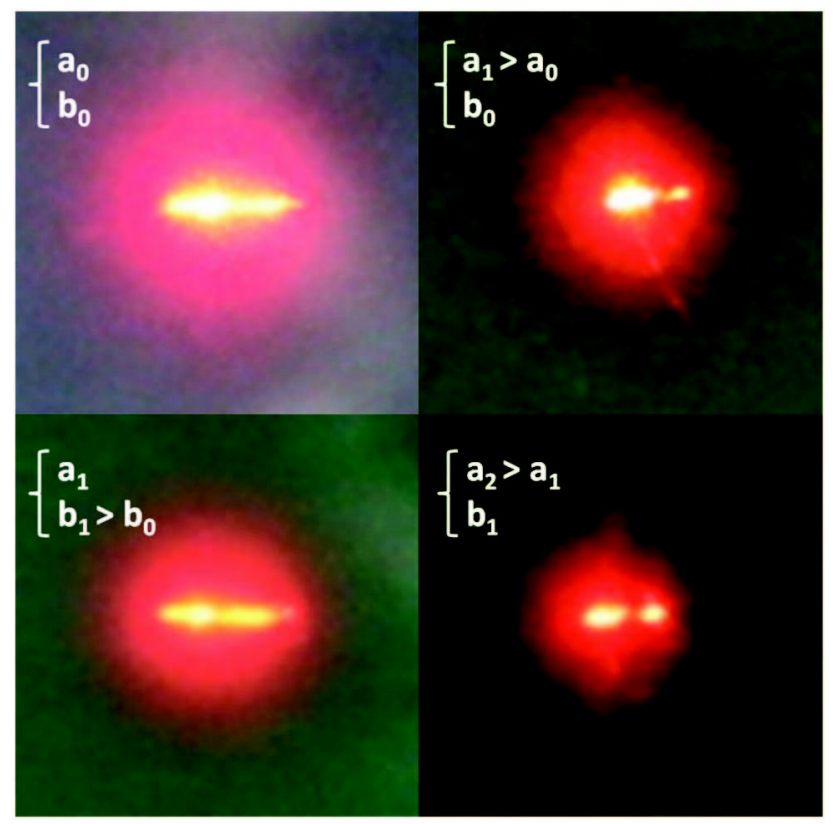

Fig. 1. Photographs of the backscattering spot of a red laser for a series of solutions with increasing concentrations of absorbing (green die) and scattering (Maalox) agents. The camera sensitivity and exposure time were held constant. The radial asymmetry is due to the noncircular laser beam used to illuminate the samples and the slight offset of the laser source and camera. scattering coefficient from $b_{0}$ to $b_{1}$ while keeping $a_{1}$ constant widens the lateral distribution of the intensity, restoring a backscattered spot size comparable to the initial case $\left(a_{0}, b_{0}\right)$. Based on the different effects of scattering and absorption on the backscattered intensity and on its lateral distribution, a similar setup that measures the backscattered light intensity as a function of distance from the center of illumination should be capable of retrieving information on both optical properties.

This measurement system would have a simple design, consisting of a collimated light source in the center of a circular photodetector. A possible application of such an instrument would be the deployment on autonomous underwater vehicles (AUVs) for routine three-dimensional profiling of seawater absorption and backscattering. While this is an exciting possibility (as, to our knowledge, it is currently impossible to measure absorption from small AUVs [8]), the focus of this paper is on the measurement concept rather than a particular application. This is a preliminary theoretical study; further adaptations and laboratory feasibility experiments will be needed to create instrument prototypes for specific applications.

\section{Methodology}

\section{A. Instrument Concept and Theoretical Approach}

We propose a simple concept for an active in situ IOP sensor (see Fig. 2). The instrument uses a monochromatic collimated light source that illuminates the water underneath it at an angle normal to the sensor. A series of concentric downlooking photodetector rings centered around the source retrieve the radial distribution of backscattered light intensity in the same wavelength as the emitted light. This measurement is normalized to the source intensity (monitored with a reference detector).

This paper presents the theoretical feasibility study for such an instrument-the first step required for its development. We use numerical modeling of radiative transfer to simulate the sensor response to optical conditions corresponding to different natural waters (the "forward problem"). The results of these simulations are analyzed to identify stable mathematical relationships between the known, imposed IOPs and some descriptors of the modeled instrument signal (see Subsection 2.C). Finally, we invert these relationships to obtain the algorithm that will be used by the instrument to convert a detected signal into estimates of the water IOPs (the "inverse problem").

\section{B. Simulation Setup}

\section{Input IOPs}

For the numerical simulations of the forward problem, we chose five different values of the particulate backscattering ratio $B_{p}: 0.025$ and 0.02 (typical for waters rich in inorganic particles $[9,10]), 0.005$ 


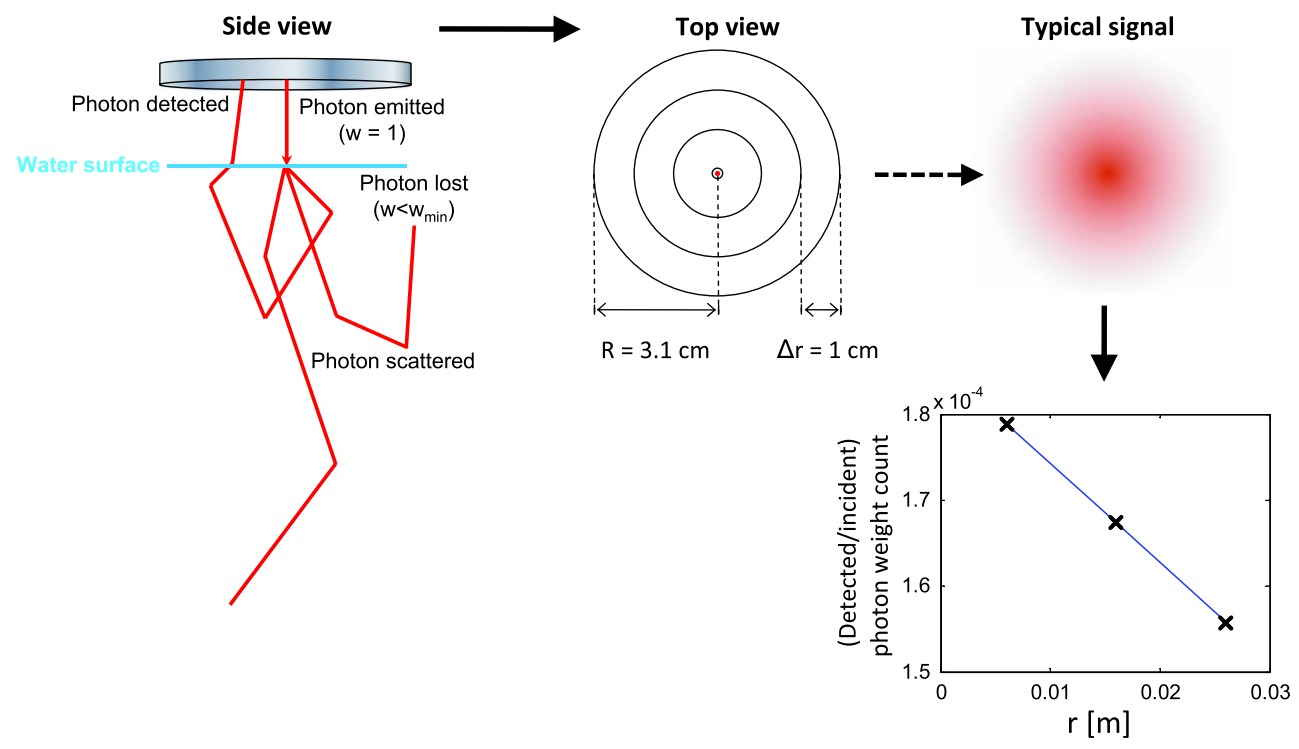

Fig. 2. Sketch of the proposed IOP sensor, with typical modeled measurement. A collimated light beam is shone into the water and the backscattered light intensity is retrieved as a function of distance from the center of illumination by three concentric photodetector rings. In the numerical simulation of the detected signal, photons are emitted with assigned weights of 1 and traced through multiple scattering events until their weights fall below a threshold $w_{\text {min }}$ or they are detected. The signal is described by the integrated photon weights within each ring as a function of the distance from the center.

(typical for waters dominated by phytoplakton, e.g., [9-12]), and 0.01 and 0.015 (intermediate values that dominate oceanic observations [13]). For each type of particle population (i.e., each value of $B_{p}$ ), we simulate the response of an instrument using red light $(\lambda=670 \mathrm{~nm})$ to 91 different combinations of optical properties $\left(a_{p}, b_{p}\right)$ (see Table 1 for notations), chosen to reflect the distribution of IOPs in natural marine environments (see Fig. 3 and Appendix A).

For each scenario, the bulk IOPs are calculated using:

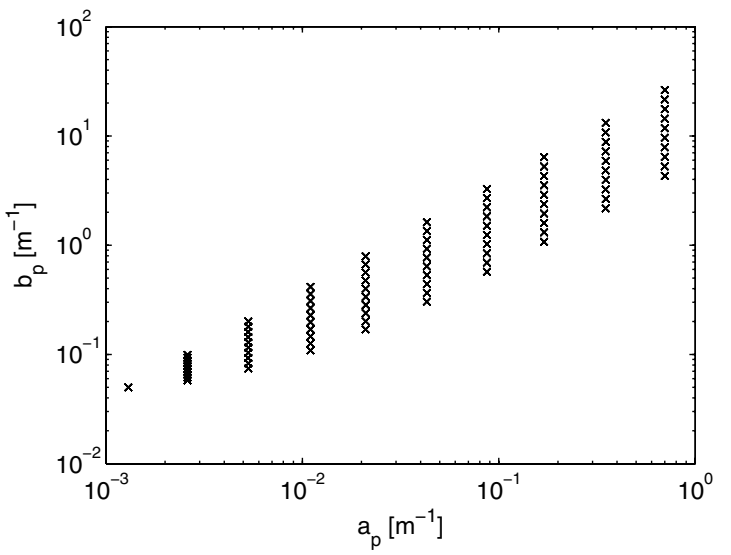

Fig. 3. Particulate absorption and scattering values chosen to drive the radiative transfer simulations used in this study $(\lambda=670 \mathrm{~nm})$. The IOP combinations are derived from the ranges of particulate absorption and scattering measured throughout a wide variety of water masses around Europe and in the North Atlantic and their relationships reported in [26,27]. These values are in agreement with measurements of $a_{p}$ and $b_{p}$ in coastal and open ocean locations around North America and the equatorial Pacific [30].

$$
\begin{gathered}
a(\lambda)=a_{s w}(\lambda)+a_{p}(\lambda)+a_{g}(\lambda), \\
b(\lambda)=b_{s w}(\lambda)+b_{p}(\lambda), \\
c(\lambda)=a(\lambda)+b(\lambda),
\end{gathered}
$$

and the seawater and gelbstoff IOPs given in Table 2.

Particle scattering is modeled using the FournierForand scattering phase function [14], a widely used analytical function to describe particle scattering in aquatic environments:

$$
\begin{aligned}
\tilde{\beta}_{F F}(\theta)= & \frac{1}{4 \pi(1-\delta)^{2} \delta^{\nu}}\left\{\nu(1-\delta)-\left(1-\delta^{\nu}\right)\right. \\
& \left.+\frac{1}{\sin ^{2}\left(\frac{\theta}{2}\right)}\left[\delta\left(1-\delta^{\nu}\right)-\nu(1-\delta)\right]\right\} \\
& +\frac{\delta_{\pi}^{\nu}-1}{16 \pi\left(1-\delta_{\pi}\right) \delta_{\pi}^{\nu}}\left(3 \cos ^{2} \theta-1\right),
\end{aligned}
$$

where

$$
\nu=\frac{3-\mu}{2}, \quad \delta=\frac{4 \sin ^{2}\left(\frac{\theta}{2}\right)}{3(n-1)^{2}}, \quad \delta_{\pi}=\delta(\pi),
$$

$n$ is the real index of refraction of the particles relative to water, and $\mu$ is the slope parameter of the power-law (Junge-type) particle size distribution. According to calculations by Mobley et al. [15], $n$ and $\mu$ can be related to the particle backscattering ratio using Eqs. (5)-(7): 
Table 1. Notations

\begin{tabular}{|c|c|c|}
\hline Variable & Units & Definition \\
\hline$a$ & $\mathrm{~m}^{-1}$ & Total absorption coefficient \\
\hline$a_{s w}$ & $\mathrm{~m}^{-1}$ & Seawater absorption coefficient \\
\hline$a_{p}$ & $\mathrm{~m}^{-1}$ & Particulate absorption coefficient \\
\hline$a_{g}$ & $\mathrm{~m}^{-1}$ & Gelbstoff absorption coefficient \\
\hline$a_{p g}$ & $\mathrm{~m}^{-1}$ & $a_{p}+a_{g}$ \\
\hline$b^{10}$ & $\mathrm{~m}^{-1}$ & Total scattering coefficient \\
\hline$b_{s w}$ & $\mathrm{~m}^{-1}$ & Seawater scattering coefficient \\
\hline$b_{p}$ & $\mathrm{~m}^{-1}$ & Particulate scattering coefficient \\
\hline$b_{b}$ & $\mathrm{~m}^{-1}$ & Total backscattering coefficient \\
\hline$b_{b s w}$ & $\mathrm{~m}^{-1}$ & Seawater backscattering coefficient \\
\hline$b_{b p}$ & $\mathrm{~m}^{-1}$ & Particulate backscattering coefficient \\
\hline$c$ & $\mathrm{~m}^{-1}$ & Total beam attenuation coefficient \\
\hline$B_{p}$ & & $\begin{array}{l}\text { Particulate backscattering } \\
\text { ratio: } b_{b p} / b_{p}\end{array}$ \\
\hline$\beta(\theta)$ & $\mathrm{m}^{-1} \mathrm{sr}^{-1}$ & VSF \\
\hline$\tilde{\beta}(\theta)$ & $\mathrm{sr}^{-1}$ & Scattering phase function \\
\hline$n_{a}$ & & $\begin{array}{l}\text { Air index of refraction (relative to } \\
\text { water) at } \lambda=670 \mathrm{~nm}\end{array}$ \\
\hline$n_{w}$ & & $\begin{array}{l}\text { Seawater index of refraction (relative } \\
\text { to water) at } \lambda=670 \mathrm{~nm}\end{array}$ \\
\hline$\lambda$ & $\mathrm{nm}$ & Wavelength \\
\hline$R$ & $\mathrm{~cm}$ & Detector radius \\
\hline$\Delta r$ & $\mathrm{~mm}$ & Thickness of detector rings \\
\hline$w$ & & $\begin{array}{l}\text { Photon weight used in radiative } \\
\text { transfer simulation }\end{array}$ \\
\hline$N(r)$ & & $\begin{array}{l}\text { Photon weight count at distance } r \\
\text { from the center }\end{array}$ \\
\hline$n(r)$ & $\mathrm{m}^{-2}$ & $\begin{array}{l}\text { Photon weight count per unit area at } \\
\text { distance } r \text { from the center in a time unit }\end{array}$ \\
\hline$C(r)$ & & $\begin{array}{l}\text { Photon weight count within distance } r \\
\text { from the center }\end{array}$ \\
\hline$N_{\text {source }}$ & & Number of photons emitted by the source \\
\hline$t$ & $\mathrm{~s}$ & Instrument exposure time \\
\hline$D$ & & Total detector photon count \\
\hline$\alpha$ & $\mathrm{m}^{-1}$ & $\begin{array}{l}\text { Signal geometry indicator (see } \\
\text { description in Subsection } \underline{2 . \mathrm{C}} \text { ) }\end{array}$ \\
\hline$\varepsilon_{a}$ & & $\begin{array}{l}\text { Relative error of } a \text { inversion: } \\
\left(a_{\text {inverted }}-a_{\text {real }}\right) / a_{\text {real }}\end{array}$ \\
\hline$\varepsilon_{\text {apg }}$ & & Relative error of $a_{p g}$ inversion \\
\hline$\varepsilon_{b b}$ & & Relative error of $b_{b}$ inversion \\
\hline$\varepsilon_{b b p}$ & & Relative error of $b_{b p}$ inversion \\
\hline
\end{tabular}

$$
\begin{gathered}
B_{p}=1-\frac{1-\delta_{\frac{\pi}{2}}^{\nu+1}-0.5\left(1-\delta_{\frac{\pi}{2}}^{\nu}\right)}{\left(1-\delta_{\frac{\pi}{2}}\right) \delta_{\frac{\pi}{2}}^{\nu}}, \quad \delta_{\frac{\pi}{2}}=\delta(\pi / 2), \\
\frac{2}{3 \delta_{\frac{\pi}{2}}}=(0.01-0.3084 \nu)^{2} .
\end{gathered}
$$

These equations are solved numerically to obtain $n$ and $\mu$ for each value of $B_{p}$, providing the particulate phase function needed for the numerical simulations.

Scattering by seawater is modeled as EinsteinSmoluchowski, or "fluctuation" scattering. The corresponding volume scattering function (VSF) is calculated following the description in [16] (Ch. 3.8):

$$
\beta_{s w}(\theta ; 670 \mathrm{~nm})=0.509\left(1+0.835 \cos ^{2} \theta\right) \cdot 10^{-4} .
$$

Finally, the resulting scattering phase function used in the Monte Carlo simulation is calculated as

$$
\tilde{\beta}=\frac{b_{p} \cdot \tilde{\beta}_{F F}+\beta_{s w}}{b_{p}+b_{s w}}
$$

\section{Radiative Transfer Model Methodology}

We use a Monte Carlo approach (see [17] for details and application examples) to simulate the forward radiative transfer problem for a simple, idealized system: an optically homogeneous, infinitely deep ocean with a flat surface (no waves). The source and detector are located parallel to the water surface, directly on top. A collimated light source emits light at $\lambda=670 \mathrm{~nm}$ perpendicular to the water surface. A circular photodetector centered around the source detects the scattered photons exiting the water within $3.1 \mathrm{~cm}$ of the incident laser beam. The instrument records the number of photons detected in each of three 1-cm-thick rings around the central $1 \mathrm{~mm}$ radius disc where the source is located.

The propagation of the light beam through the airwater interface is modeled according to Snell's law and Fresnel's formula, using the indices of refraction for water $n_{w}=1$ and air $n_{a}=0.75$ [16].

To maximize the efficiency of the numerical simulation, we use photon weighting (see Chapter 2.3 in [17]), a technique that minimizes loss of computational effort due to tracing of photons that are not of interest (photons that do not reach the detector). In the model, the source emits photon packets, rather than individual photons. Each emitted photon packet has an initial weight $w=1$. This weight decreases with each event of scattering or internal reflection off the water-air interface by the probability that absorption took place. The photon packet is traced until its weight reaches a minimum threshold $\left(10^{-6}\right)$ or it is detected by the IOP sensor.

Photons are emitted until the detector records a predefined number of backscattered photons $(30,000)$, chosen to minimize the Monte Carlo noise, while still allowing for reasonable computation times. At the end of the simulation, the signal is normalized by the number of photons generated to create it.

The numerical simulations were carried out using the MATLAB programming language on a computing cluster with variable processing time, ranging from one to four weeks per run on a $2 \mathrm{GHz}$ Intel processor.

The MATLAB codes for the radiative transfer model, the data analysis routines, and the final inversion

Table 2. Constant IOP Values Used in the Optical Model $(\lambda=670 \mathrm{~nm})$

\begin{tabular}{lcc}
\hline IOP & Value & Source \\
\hline$a_{s w}$ & $0.43 \mathrm{~m}^{-1}$ & {$[\underline{16}]$} \\
$a_{g}$ & $\sim 0 \mathrm{~m}^{-1}$ & {$[\underline{31}, \underline{30}]$} \\
$b_{s w}$ & $8.176 \times 10^{-4} \mathrm{~m}^{-1}$ & {$[\overline{16}]$} \\
$n_{a}$ & 0.75 & {$[\underline{16}]$} \\
$n_{w}$ & 1 & $\underline{\underline{16}}]$ \\
\hline
\end{tabular}


algorithm are available at http://misclab.umeoce .maine.edu/code/IOP_inversion_model/. The model runs with user-defined light beam incidence angle, target number of detected photon packets, maximum number of scattering events per photon packet and minimum photon packet weight, detector radius and resolution, environment index of refraction and backscattering ratio. The code can easily be adapted to simulate environments with different absorption and scattering properties, and different instrument locations with respect to the water surface (immersed or at any height above the water).

\section{Data Analysis}

For each experiment (each set of IOPs $-B_{p}, a_{p}, b_{p}$ ), the model output is a function $N(r)$ of the sum of all photon weights detected at a distance $r$ from the center of illumination:

$$
N(r)=\frac{1}{N_{\text {source }}} 2 \pi \int_{0}^{t} \int_{r-\frac{\Delta r}{2}}^{r+\frac{\Delta r}{2}} n\left(r^{\prime}\right) r^{\prime} \mathrm{d} r^{\prime} \mathrm{d} t^{\prime}
$$

where $N_{\text {source }}$ is the number of photon packets emitted to obtain the signal, $\Delta r$ is the ring detector width $(\Delta r=1 \mathrm{~cm}), n(r)$ is the sum of photon weights detected per unit area at distance $r$ from the center in a time unit, and $t$ is the detector's exposure time.

We synthesize this model output into two parameters, one describing the total amount of backscattered light detected by the instrument $(D)$ and one describing the geometry of the signal $(\alpha)$.

$D$ is calculated by integrating the photon weights detected over all the rings, except for the central $1 \mathrm{~mm}$ radius disc [Eq. (11)].

$$
D=\frac{1}{N_{\text {source }}} 2 \pi \int_{0}^{t} \int_{1 \mathrm{~mm}}^{R} n\left(r^{\prime}\right) r^{\prime} \mathrm{d} r^{\prime} \mathrm{d} t^{\prime},
$$

where $R=3.1 \mathrm{~cm}$ is the instrument radius. This region is masked out, as it corresponds to the location of the source light beam.

$\alpha$ is calculated as the slope $\frac{d C}{d r}$ of the angular cumulative backscattered photon weight count $C(r)$ [Eq. (12)] in a region close to the center of the detector. Because within $3.1 \mathrm{~cm}$ around the source this function is roughly linear (Fig. 4), we calculate $\alpha$ from a linear fit to $C(r)$ within a radius of $3.1 \mathrm{~cm}$. This parameter can be interpreted as an indicator of the lateral spread of the detected light intensity:

$$
C(r)=\frac{1}{N_{\text {source }}} 2 \pi \int_{0}^{t} \int_{1 \mathrm{~mm}}^{r} n\left(r^{\prime}\right) r^{\prime} \mathrm{d} r^{\prime} \mathrm{d} t^{\prime}
$$

\section{Results}

An analysis of the data set obtained from the model runs covering the entire domain of IOP values revealed two stable relationships between the signal descriptors and the water optical properties (see

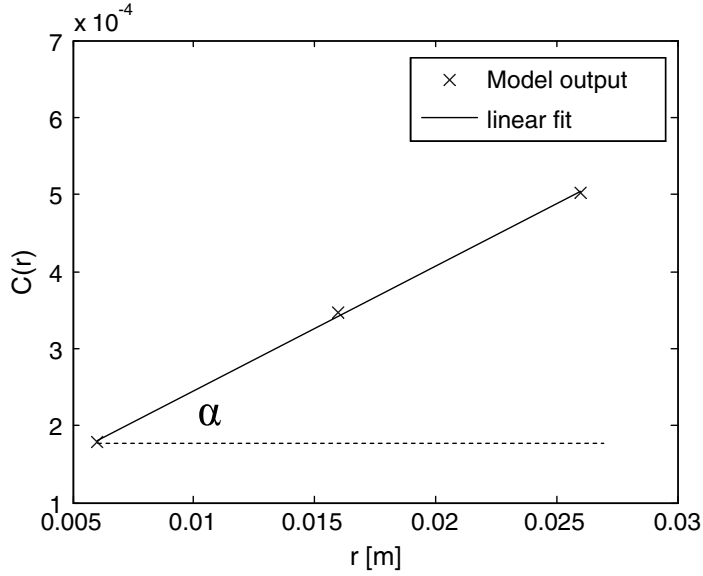

Fig. 4. Graphical representation of the calculation of $\alpha$ for a sample data set $\left(B_{p}=0.01, a_{p}=0.17 \mathrm{~m}^{-1}, b_{p}=2.9 \mathrm{~m}^{-1}\right)$.

Fig. 5). The total backscattering coefficient is best related to the geometry descriptor $\alpha$ through a linear relationship in log-log space:

$$
b_{b}=10^{1.048 \log (\alpha)+0.3409} .
$$

We also find a well-constrained relationship between the ratio of the backscattering and absorption coefficients and the total retrieved photon packet count, which we fit a second-order polynomial in $\log -\log$ space to:

$$
\frac{b_{b}}{a}=10^{-0.07407 \log ^{2}(D)+0.3525 \log (D)+0.6555} .
$$

Equations (13) and (14) represent empirical relationships and can be used to obtain $a$ and $b_{b}$ from a given measurement described by $D$ and $\alpha$, within the IOP range considered (Subsection 2.B.1). The resulting distribution of relative errors when this inversion is applied to the original data set is shown in Fig. 6 .

The relative errors do not exceed $13.4 \%$ for the inversion of $b_{b}$ and $56.9 \%$ for the inversion of $a$, with $90 \%$ of the errors below $6.9 \%$ for $b_{b}$ and below $29.7 \%$ for $a$. An investigation of the distribution of these errors reveals a relationship between the inversion uncertainties for both IOPs and the value of the total single-scattering albedo $b / c$. The higher absolute values of the relative errors of inversion typically correspond to higher values of the singlescattering albedo, with the inversion for waters with $b / c<0.7$ resulting in relative errors less than $10 \%$ for $b_{b}$ and less than $30 \%$ for $a$ (Fig. 7).

These errors compare well with theoretical errors for current instruments typically used to measure the two IOPs: Boss et al. [10] report an estimated $10 \%$ error associated with the correction scheme they describe for $b_{b}$ measurements with the HOBILabs Hydroscat-6 [18]; an error of a few percent (up to 10\%) can be inferred from [19], which evaluates the widely used methodology of calculating $b_{b}$ from the VSF measurement at a single angle in the 


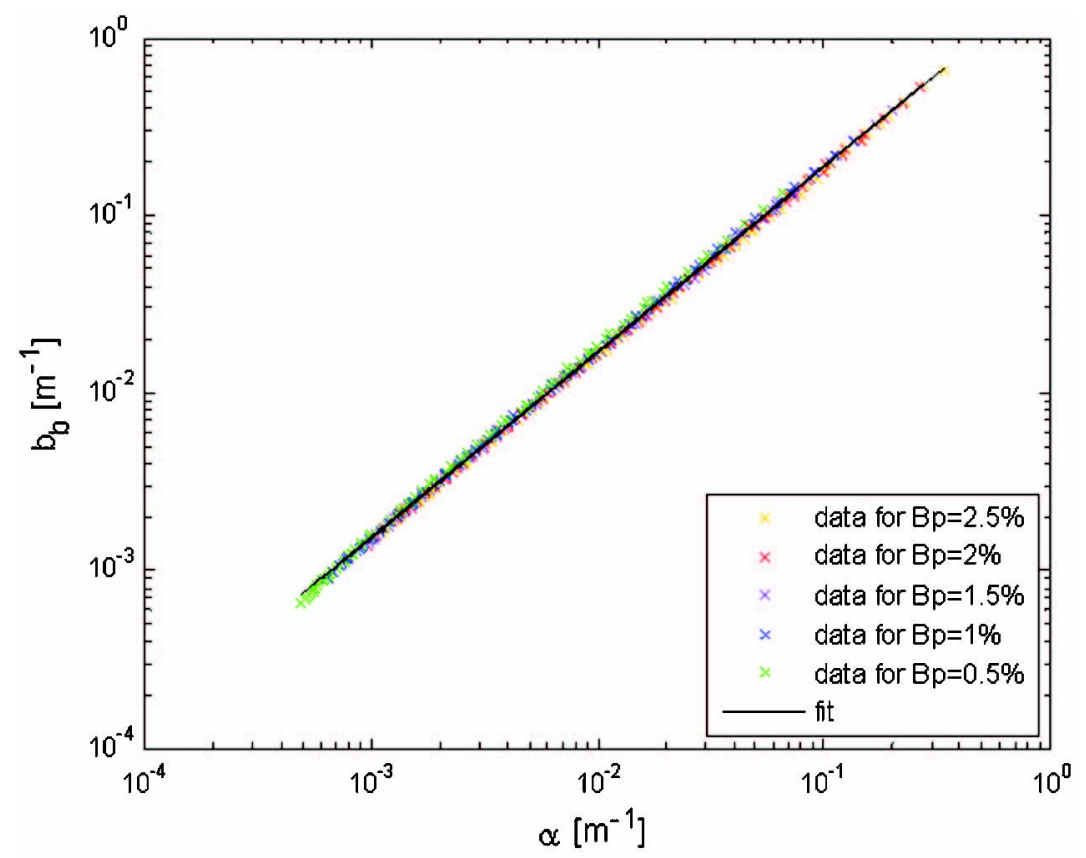

(a)

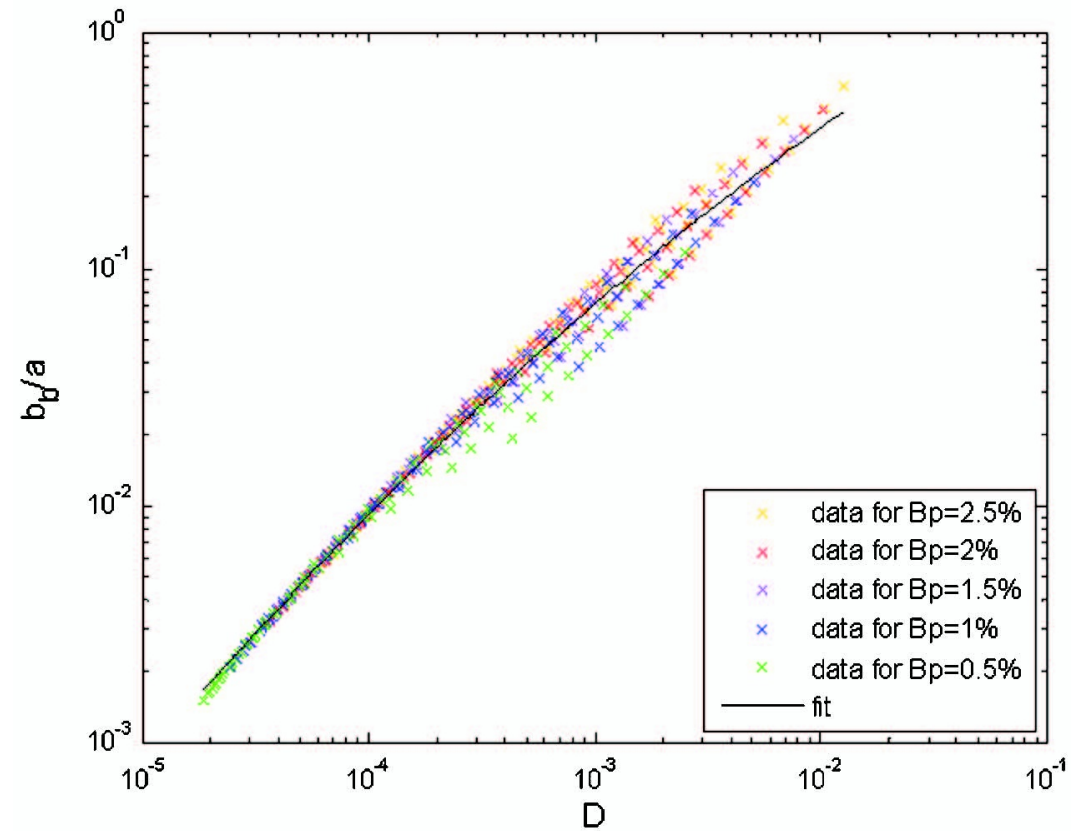

(b)

Fig. 5. Water IOPs plotted against resulting signal descriptors: (a) backscattering coefficient versus geometry parameter $\alpha$, (b) ratio between backscattering and absorption versus detected photon count $D$. The different colors correspond to different particle backscattering ratios, and the black lines correspond to Eqs. (13) and (14), which numerically fit the data.

backward direction. A recent Monte Carlo study [20] calculates the theoretical uncertainties in IOP measurements with the WET Labs AC-9 spectrophotometer [21] and shows errors of up to $20 \%, 75 \%$, or $140 \%$, depending on the water type, for measurements of $a$ at $670 \mathrm{~nm}$.

Note that the particle backscattering ratio has little effect on the relationships shown in Fig. 5; thus, they can be used to invert for $b_{b}$ and $a$ without prior knowledge of the water type (to the extent to which the range of IOPs considered here is representative of the environment).

\section{Discussion}

\section{A. IOP Inversions}

\section{Sensitivity to $B_{p}$}

While most investigators performing IOP measurements in natural waters have found $B_{p}$ values 


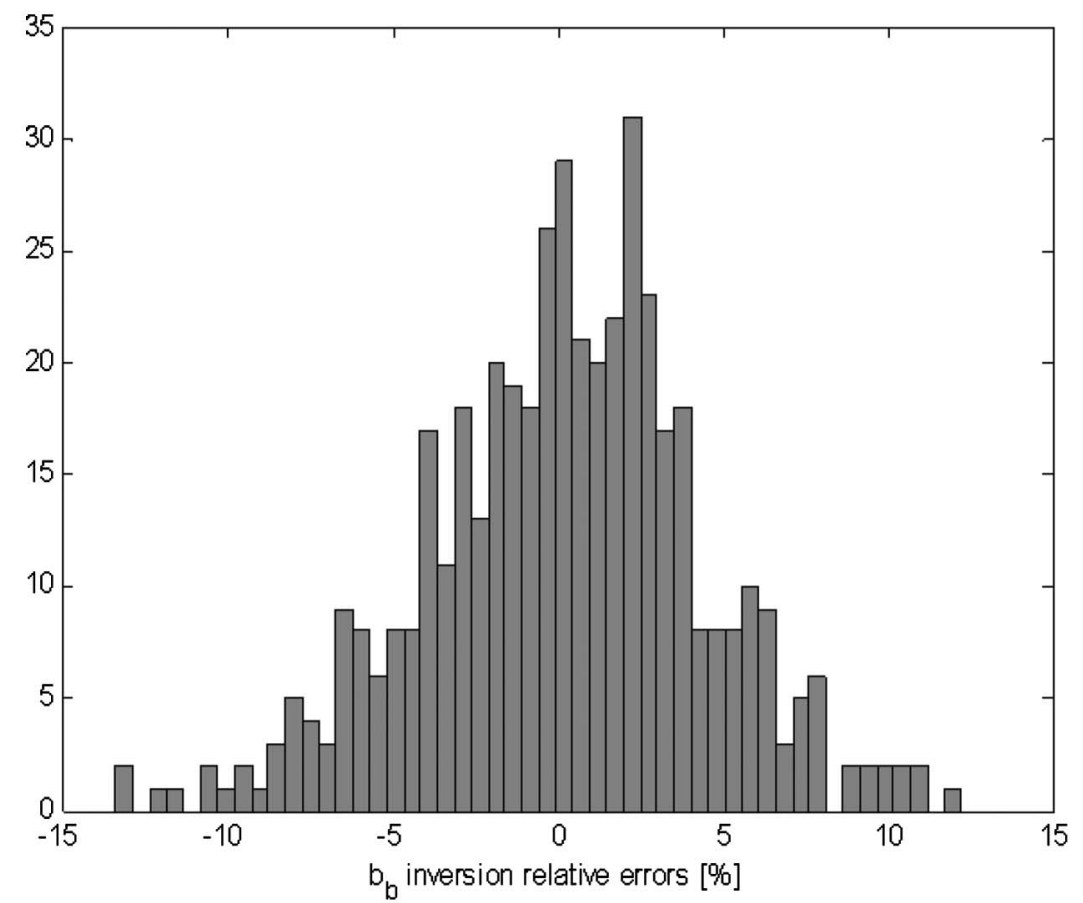

(a)

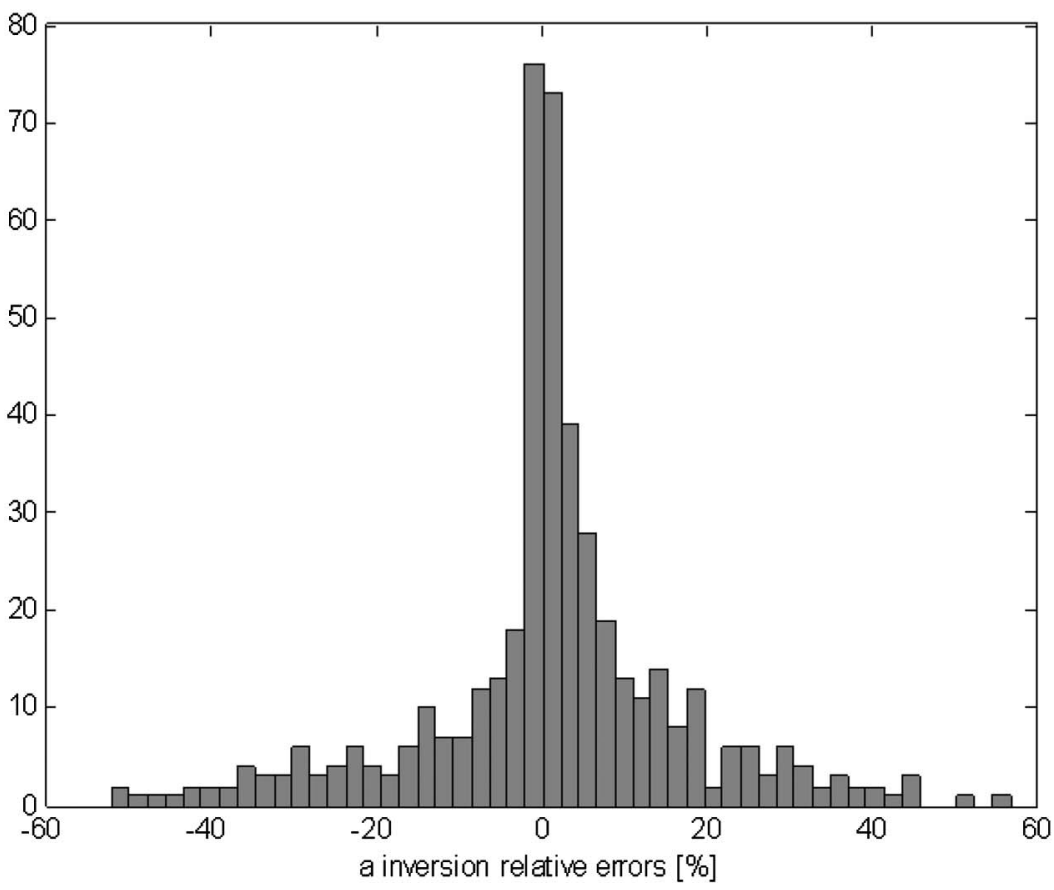

(b)

Fig. 6. Histograms of the relative errors in inverting (a) $b_{b}$ and (b) $a$ from modeled instrument response to full range of IOPs.

within the range considered in our simulations, higher particle backscattering ratios have also been reported for mineral-rich coastal waters (e.g., [22]). To test the sensitivity of our inversion algorithm to possible extreme values of $B_{p}$, we ran the Monte Carlo optical model for the same set of particle absorption and scattering coefficients presented in Fig. 3 with a particle backscattering ratio value of $5 \%$, and we used the inversion algorithm to retrieve the values of $a$ and $b_{b}$ for each numerical experiment. In $90 \%$ of the cases, the backscattering coefficient was inverted with relative errors below $15.2 \%$ and the absorption coefficient was inverted with relative errors within $68.1 \%$, with both $b_{b}$ and $a$ being consistently overestimated. However, if we include this data set in the regressions we use to determine the inversion equations, we only slightly degrade the performance of the inversion algorithm for all 
Table 3. Inversion Errors for Simulated Detector Responses to Entire Range of IOP Combinations for Different Inversion Algorithms, Obtained with the "Training Data Sets" and Applied on the "Test Data Sets"

\begin{tabular}{|c|c|c|c|c|c|}
\hline $\begin{array}{l}B_{p}(\%) \\
\text { Training Data Set }\end{array}$ & $\begin{array}{c}B_{p}(\%) \\
\text { Test Data Set }\end{array}$ & $\begin{array}{c}\max \left|\varepsilon_{b b}\right|(\%) \\
(\text { All Data })\end{array}$ & $\begin{array}{c}\max \left|\varepsilon_{b b}\right|(\%) \\
(90 \% \text { of Data })\end{array}$ & $\begin{array}{c}\max \left|\varepsilon_{a}\right|(\%) \\
(\text { All Data })\end{array}$ & $\begin{array}{c}\max \left|\varepsilon_{a}\right|(\%) \\
(90 \% \text { of Data })\end{array}$ \\
\hline $0.5-2.5$ & $0.5-2.5$ & 13.4 & 6.9 & 56.9 & 29.7 \\
\hline $0.5-2.5$ & 5 & 18.2 & 15.1 & 95.9 & 68.0 \\
\hline $0.5-5$ & $0.5-5$ & 15.0 & 8.9 & 66.3 & 31.5 \\
\hline 0.5 & 0.5 & 8.7 & 5.5 & 40.0 & 25.7 \\
\hline 1 & 1 & 6.8 & 3.8 & 39.1 & 23.2 \\
\hline 1.5 & 1.5 & 6.1 & 3.7 & 38.2 & 23.0 \\
\hline 2 & 2 & 6.3 & 4.3 & 38.8 & 22.9 \\
\hline 2.5 & 2.5 & 8.0 & 5.4 & 38.7 & 24.1 \\
\hline 5 & 5 & 9.3 & 5.6 & 37.6 & 22.7 \\
\hline
\end{tabular}

the numerical experiments compared to the original results presented in Section 3 (see the first part of Table 3).

Note that, within these error ranges, this inversion algorithm performs best for experiments with $B_{p}$ between $1 \%$ and $1.5 \%$ (corresponding to the most frequent particle backscattering ratios in a wide range of aquatic environments [13,23]), with the error ranges increasing for higher (and, in the case of $b_{b}$, also lower) $B_{p}$ values (see Fig. 7). These results once again compare well with theoretical error calculations for the measurement of the absorption coefficient with WETLabs AC-9 instrumentation [20], which show a significant error increase in waters dominated by inorganic particles (compared to chlorophyll-dominated or mixed waters), especially in high scattering regimes. Note that the errors reported in [20] are up to approximately $140 \%$ for mineral-rich waters at $\lambda=670 \mathrm{~nm}$ by comparison to our worst-case scenario where the maximum obtained error for $a$ is below $96 \%$ (see the second line in Table 3).

The aforementioned tests show that the type of inversion algorithm we propose is sensitive to the IOP ranges used to generate it. While the inversion we exemplify in Section 3 (based on a wide range of IOPs encompassing the bulk of natural waters) results in error ranges comparable to similar theoretical uncertainty estimates for classic IOP sensors, a better knowledge of the environment would help constrain the inversion algorithm, resulting in better estimates of the backscattering and absorption coefficients. As shown in the second part of Table 3, if $B_{p}$ is known (e.g., from having concurrent beam attenuation measurements), the inversion can be tuned to return estimates of $a$ within less than $40 \%$ and $b_{b}$ within a maximum relative error of approximately $8 \%$ to $9 \%$ for either inorganic particle or chlorophyll-dominated waters and approximately $6 \%$ for intermediate waters.

\section{Sensitivity to VSF}

To further explore the sensitivity of our inversion algorithm to the VSF, we tested it on data from simulations where particle scattering was described by the empirical Petzold average-particle phase function [16]. We find that, although the inversion algorithm is trained with data from Fournier-Forand simulations, it performs equally well on data based on Petzold scattering (see Appendix B.1). We conclude that the inversion we obtained for $a$ and $b_{b}$ is not significantly sensitive to details of scattering in the back direction. To the extent to which the variability in the shape of the VSF included in this study (see Fig. 8) is representative of the range of VSF shapes in natural waters, this inversion can be used with equal confidence on different water types.

\section{Bulk Versus Component-Specific IOPs}

For the use of IOPs to invert biogeochemical properties of interest, it is only the variable contributions of dissolved and particulate constituents in the water that are important, as opposed to the known and constant (for the same temperature-salinity conditions) contribution of pure seawater to the bulk IOPs:

$$
\begin{gathered}
b_{b p}=b_{b}-b_{b s w}, \\
a_{p g}=a-a_{s w} .
\end{gathered}
$$

Our ability to invert for the contributions of individual components to absorption and backscattering depends on the ability to estimate the bulk IOPs $\left(a\right.$ and $b_{b}$ ) and the weight of each component on these bulk properties [see Eqs. (17) and (18)]:

$$
\begin{gathered}
\varepsilon_{a p g}=\frac{a}{a_{p g}} \cdot \varepsilon_{a}, \\
\varepsilon_{b b p}=\frac{b_{b}}{b_{b p}} \cdot \varepsilon_{b b},
\end{gathered}
$$

where $\varepsilon_{x}$ denotes the relative error in retrieving property $x$.

Because water, particulate, and dissolved matter weigh differently on $a$ and $b_{b}$ at different wavelengths, some knowledge of these typical relationships in natural waters will have to be employed in deciding on the use of a specific wavelength for the 


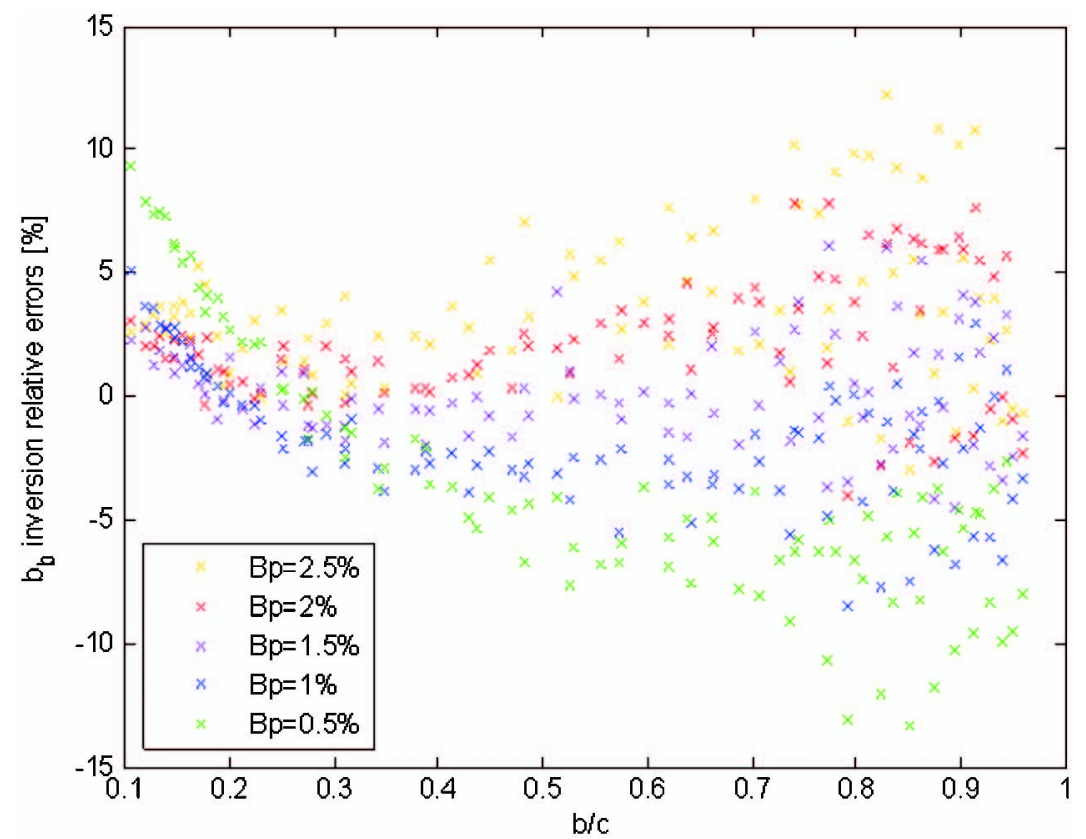

(a)

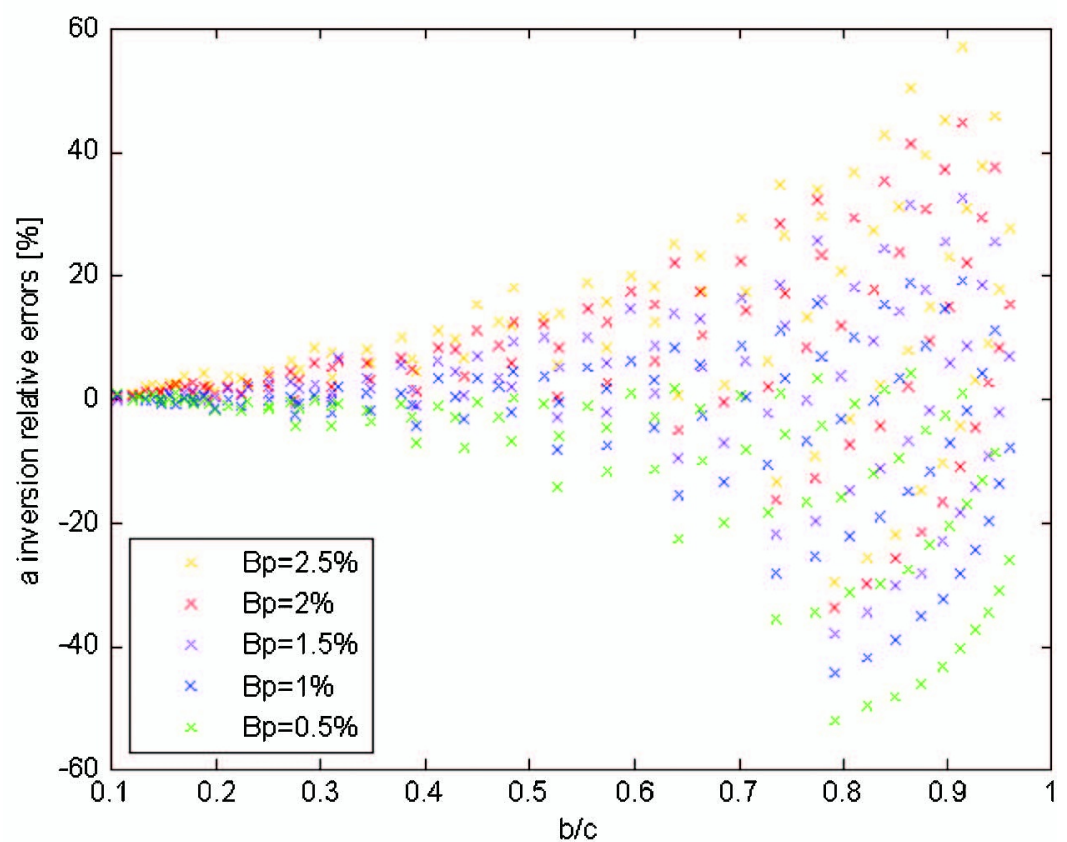

(b)

Fig. 7. Relative errors in inverting (a) $b_{b}$ and (b) $a$ plotted against the corresponding total single-scattering albedo values $b / c$. The different colors indicate numerical experiments with different particle backscattering ratios.

envisioned application. For example, at $670 \mathrm{~nm}$, backscattering by particles dominates $b_{b}$, and, from our simulations, $90 \%$ of the model $b_{b p}$ values are predicted within less than $7.9 \%$ relative difference. However, at this wavelength, absorption by pure seawater is very important and, in some cases, corresponding to very high single-scattering albedos in mineral particle-dominated waters, $a_{p}$ is inverted with relative errors as large as $700 \%$ (the errors fall below $300 \%$ for $90 \%$ of the data, including all simulations of phytoplankton-dominated waters). These errors will significantly decrease for an instrument using blue light, as water absorption in this case would be much weaker. Many IOP measurements already make common use of multiple wavelength sources. An instrument using a source with several specific wavelengths would offer more information and constrain the inversion of component-specific IOPs, as is done, for example, in the inversion of satellite ocean color.

\section{B. Sensitivity to Sensor Characteristics}

Practical considerations related to instrument manufacturing and envisioned use could impose 


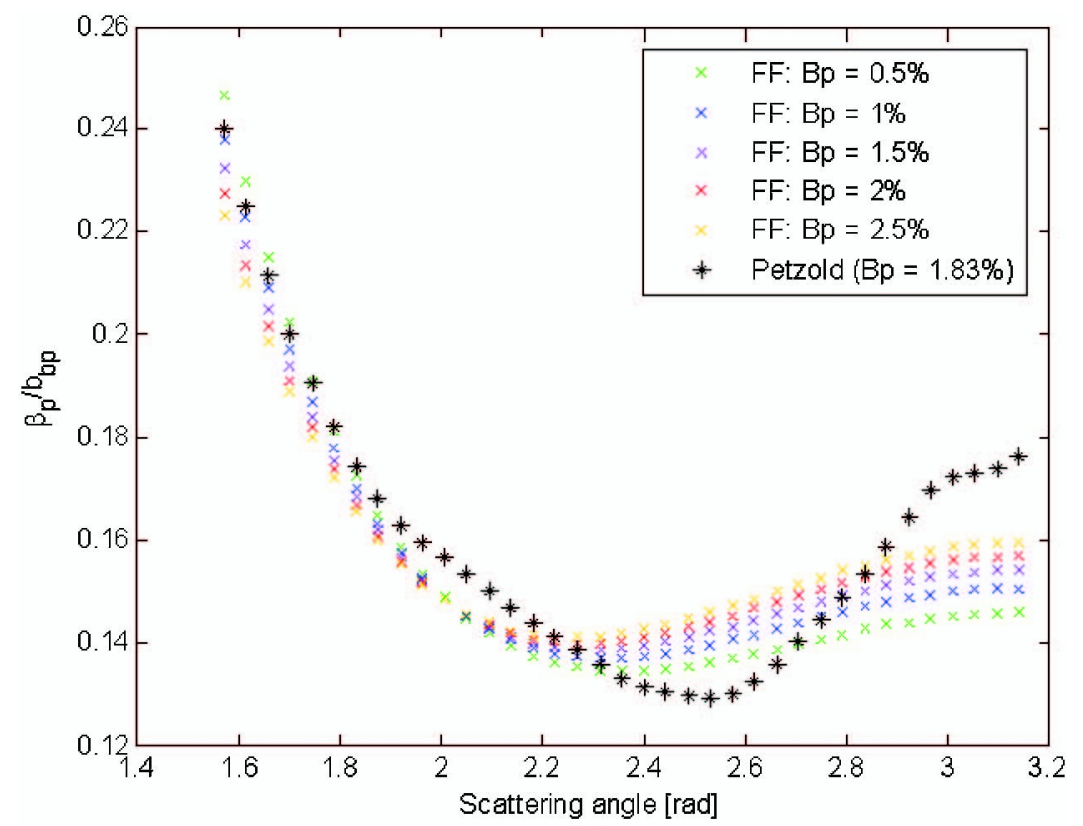

(a)

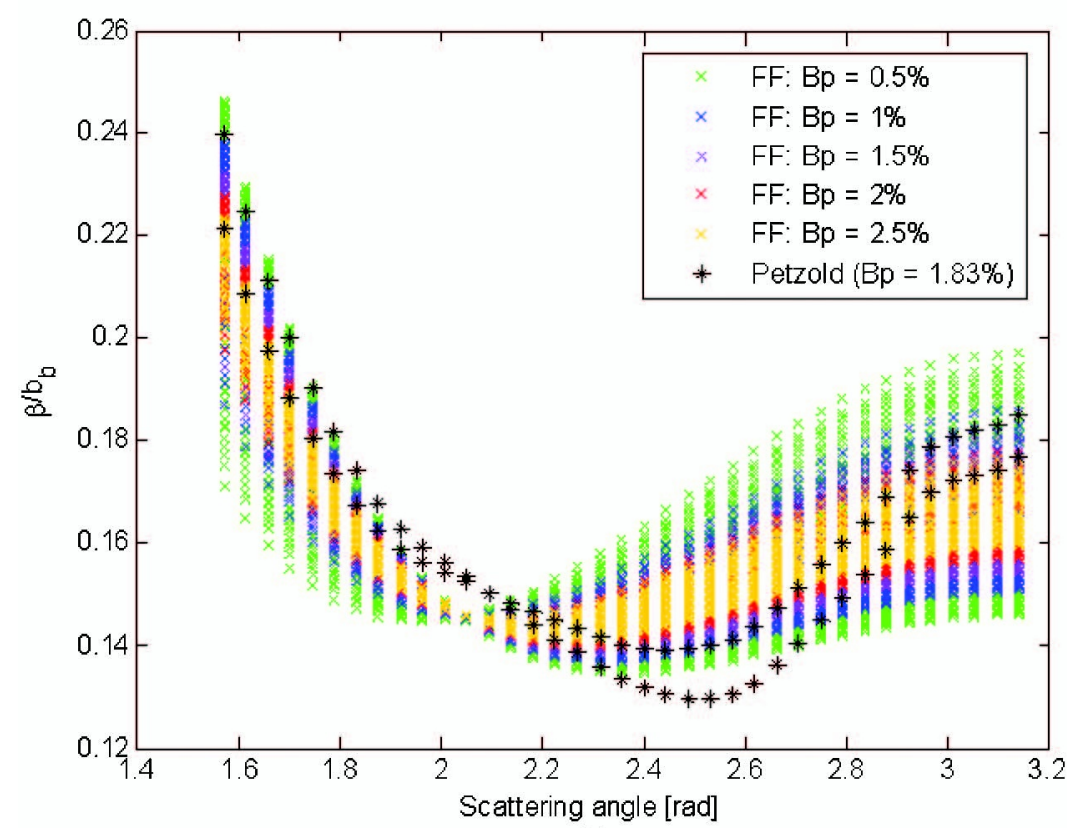

(b)

Fig. 8. Range of shapes of the VSF in the back direction used in this study. (a) Particulate VSF normalized by the particulate backscattering coefficient, (b) total VSF normalized by the total backscattering coefficient. FF stands for Fournier-Forand.

constraints on the sensor specifications (detector size and resolution, source wavelength, instrument casing, etc.). The methodology presented here can be applied for other instrumental configurations (and the optical model can easily be adapted to any such specifications), however, the quality of the obtained IOP inversion could be sensitive to some of these parameters.

To test for the sensitivity of the signal and associated inversion algorithm performance to the detector size, we ran our entire simulation suite with a maximum detector radius of $1 \mathrm{~m}$ and analyzed the data collected within $3.1,4,10$, and $100 \mathrm{~cm}$ of the source. We created an inversion algorithm for each of these four data sets and compared the obtained ranges of relative errors in the retrieval of $a$ and $b_{b}$. The inversion of the backscattering coefficient is based on a linear fit to the cumulative detected photon count $C(r)$ (see Subsection 2.C). Since $C(r)$ is only approximately linear in a region close to the center, deviating from it at distances larger than a few centimeters from the source (see Fig. 9), $b_{b}$ is inverted using the information from the first $3.1 \mathrm{~cm}$ around the center only, and, consequently, the quality of the inversion does not change with an increase in the instrument size. 


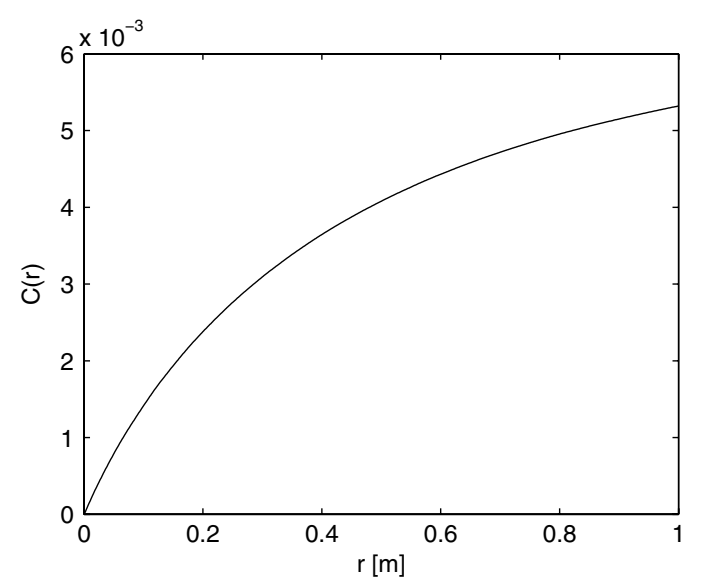

Fig. 9. Typical cumulative detected photon weight count for a $1 \mathrm{~m}$ radius sensor (sample data set obtained with input IOPs: $B_{p}=0.01, a_{p}=0.17 \mathrm{~m}^{-1}, b_{p}=2.9 \mathrm{~m}^{-1}$ ).

The absorption coefficient, however, is determined based on the amount of light retrieved by the entire detector [Eqs. (14) and (13)]. Our test shows that the inversion algorithm for $a$ performs better when given information from a larger detector (see Table 4): the maximum relative error in inverting $a$ was $28.6 \%$ (with $90 \%$ of the data inverted with relative errors less than $13.7 \%$ ) when using a $2 \mathrm{~m}$ diameter sensor, while a $6.2 \mathrm{~cm}$ diameter instrument provided information to predict $a$ within as much as $56.9 \%$ relative error (29.7\% for $90 \%$ of the data). These results indicate that, while most information on the backscattering coefficient can be retrieved from an area near the source of illumination, the signature of absorption stretches over a larger area and, depending on the scattering characteristics of the water, there may be significant information on $a$ to be retrieved from distances larger than a few centimeters from the source. This sensitivity should be taken into consideration when designing an instrument aimed at a specific application, as a compromise will be needed between compactness and accuracy of absorption retrieval.

While the instrument size has an effect on the quality of the inversion of the absorption coefficient, it is plausible that the inversion of the backscattering coefficient, based on the geometry of the signal around the center, could depend on the detector resolution $(\Delta r)$. To test for this effect, we performed another series of simulations where the detector spacing was $\Delta r=1 \mathrm{~mm}$ (while keeping the detector

\begin{tabular}{lcc}
$\begin{array}{c}\text { Table 4. } \\
\text { Response of Detectors of Different Sizes to Entire Range of IOP } \\
\text { Combinations }\end{array}$ \\
\hline $\begin{array}{l}\text { Detector Radius } \\
(\mathrm{cm})\end{array}$ & $\begin{array}{r}\max \left|\varepsilon_{a}\right|(\%) \\
(\text { All Data })\end{array}$ & $\begin{array}{c}\max \left|\varepsilon_{a}\right|(\%) \\
(90 \% \text { of Data })\end{array}$ \\
\hline 3.1 & 56.9 & 29.7 \\
4 & 55.7 & 29.1 \\
10 & 52.2 & 26.5 \\
100 & 28.6 & 13.7 \\
\hline
\end{tabular}

\begin{tabular}{lcc}
$\begin{array}{c}\text { Table 5. Inversion Algorithm Performance on Simulated } \\
\text { Response of Detectors of Different Resolutions to Entire Range of } \\
\text { IOP Combinations }\end{array}$ & \\
\hline $\begin{array}{l}\text { Detector Resolution } \\
(\mathrm{mm})\end{array}$ & $\begin{array}{c}\max \left|\varepsilon_{b b}\right|(\%) \\
(\text { All Data })\end{array}$ & $\begin{array}{c}\max \left|\varepsilon_{b b}\right|(\%) \\
(90 \% \text { of Data })\end{array}$ \\
\hline 10 & $13.4 \%$ & 6.9 \\
1 & $12.1 \%$ & 6.6 \\
\hline & $\max \left|\varepsilon_{a}\right|(\%)$ & $\max \left|\varepsilon_{a}\right|(\%)$ \\
& $($ All Data $)$ & $(90 \%$ of data $)$ \\
\hline 10 & 56.9 & 29.7 \\
1 & 54.6 & 29.0 \\
\hline
\end{tabular}

radius to its standard value of $3.1 \mathrm{~cm}$ ). A comparison of the modeled IOP inversion quality for simulated instruments with detector resolutions of $1 \mathrm{~mm}$ and $1 \mathrm{~cm}$, respectively, is given in Table 5. Although a change in the detector resolution does not affect the calculation of $D$, it does affect the inversion of $a$ indirectly, through the inverted $b_{b}$ values used in Eq. (14) to obtain $a$.

These results show that a significant (tenfold) increase in the instrument resolution would yield an almost negligible improvement in the IOP measurements (less than 2\%), rendering the effort to obtain a resolution finer than $1 \mathrm{~cm}$ practically worthless.

Another technical parameter that needs to be considered is the laser beam thickness. The model used here idealizes the source, simulating it as infinitesimally thin; however, a true laser beam has a nonnegligible width. This has the same effect on the instrument retrieval as superimposing multiple slightly offset images of the idealized modeled signal. This is not expected to modify the $\alpha$ values, but should have a small effect on $D$. Thus, while we expect the inversion for $b_{b}$ to remain the same, the inversion relationship for $a$ will have some dependence on the size of the light source.

Several auxiliary elements could be added to the instrument for measurement optimization. To ensure a smooth signal and to eliminate errors caused by source intensity drift with time, the instrument should include a reference detector to monitor the source strength, and it should be programmed to stop the measurement only once a preset threshold in the number of detected photons has been reached. This value should be normalized to the emitted photon count corresponding to each measurement. To remove ambient light effects, each measurement could be corrected with information from a background measurement without the activated source and with the same time exposure as the active measurement. Finally, it should be noted that the optical model used here simulates the propagation of unpolarized light, chosen for simplicity and because the azimuthally integrated signal is not sensitive to polarization. However, polarized light (e.g., a laser beam) may interact differently with the different components of the water, and the inversion relationships presented here may not be applicable to noncircular instruments using a polarized light source. To eliminate 
polarization-related effects, the source could be equipped with a depolarizer, providing an unpolarized beam.

\section{Deployment Setup and Applications}

The radial symmetry of the signal retrieved by the IOP sensor (using a circular light beam) permits many variations in the instrument shape, so the system we propose for measuring $a$ and $b_{b}$ could be mounted on a variety of platforms. One exciting option is the deployment of such a system on autonomous and Lagrangian platforms and sensors (ALPS) [24]. While some small AUVs are already equipped with backscattering sensors [25], we are not aware of any absorption measurements from this type of platform up to the present.

The set of numerical simulations that yielded Eqs. (13) and (14) considered an instrument located on the water surface. In the case of a $b_{b}$ and $a$ sensor affixed to an AUV, the instrument would be submerged. We tested the theoretical sensitivity of the inversion algorithm to the vicinity of the instrument to the reflective water surface and found that it only introduces minor variations in the detected signal and that the previously obtained IOP inversion algorithm performs equally well on data from a deeply submerged instrument as on data from an on-water sensor that was used in the training of the algorithm (see Appendix B.2). Note that, if the sensor is fixed on the underside of the glider (downlooking), the AUV surface might act similarly to the reflective waterair boundary in our original simulations. To ensure a minimum effect on the measurements, the underside of the AUV could be coated with black, light-absorbing paint.

The sensor type we propose may not have to be limited to in-water applications. The same methodology can be used to test the feasibility of a dry, downlooking water IOP measurement system, mounted above the surface (e.g., on a dock or under a bridge). By minimizing biofouling, such an instrument would be ideal for long-term monitoring of water optical properties. However, this application would require several (some, nontrivial) adjustments to the radiative transfer model and data analysis routines to account for the possibly varying distance between the water surface and the instrument, the sea surface roughness, and the signal contamination with specular reflection off the water surface. Along with the software package necessary to develop and assess the IOP inversion algorithm for a given system, our web link also provides a list of challenges associated to the design of an above-water instrument and some ideas for overcoming them.

\section{Conclusions}

We propose a concept for a simple, compact, active in situ IOP sensor, capable of simultaneously providing an estimate of the absorption $(a)$ and backscattering $\left(b_{b}\right)$ coefficients of natural water. We test the feasibility of such a system using Monte Carlo simulations of light propagation through waters with different optical characteristics, spanning the bulk of the observed range of natural values. We isolate two mathematical relationships between the descriptors of the modeled instrument signal and $a$ and $b_{b}$. These relationships form the general inversion algorithm our IOP measurement system would use to convert a detected signal into estimates of $a$ and $b_{b}$, without a priori knowledge of the water components. However, when available, concurrent measurements of other IOPs could be employed to constrain the IOP range used with our methodology to generate an application-optimized inversion algorithm and consequently obtain better estimates of the backscattering and absorption coefficients. We find the uncertainties of this sensor concept, when applied to the specific wavelength of $670 \mathrm{~nm}$, to be of the same order and slightly better than current commercial instrumentation.

Our modeling results indicate that the new instrument can be designed to have different geometries and to use different wavelengths, depending on the envisioned applications, and our optical simulation (which we make available online) can easily be adapted to describe different setups. This study opens multiple directions of investigation and possible applications, including ALPS-mounted $a$ and $b_{b}$ sensors and possibly dry in situ instrumentation to measure absorption and backscattering (ideal for long-term monitoring of water IOPs).

\section{Appendix A: Choice of IOPs to Drive Optical Simulations}

The range of particulate absorption values was derived from the results of six Coastal Surveillance Through Observation of Ocean Color sampling campaigns and additional optical measurements in open ocean waters in the North Atlantic presented in [26]. Figure 15 in [26] provides the ranges of absorption coefficients by nonalgal particles at $443 \mathrm{~nm}$ $\left(a_{\mathrm{NAP}}(443)\right)$ in the six different major sampling locations. These values are converted to absorption coefficients at $670 \mathrm{~nm}$ using Eq. (4) and the $S_{\mathrm{NAP}}$ values for each location given in Table 4 in [26]. Furthermore, Fig. 7 in the same paper provides the range for the algal particulate absorption coefficients at $676 \mathrm{~nm}\left(a_{\phi}(676)\right)$, a fair indication for the range of $a_{\phi}(670)$. Finally, we obtain the particulate absorption coefficient by adding the algal and nonalgal components:

$$
a_{p}(670)=a_{\phi}(670)+a_{\mathrm{NAP}}(670) .
$$

The particulate scattering coefficients measured during the same sampling campaigns are presented in [27]. We considered the range of particulate scattering coefficients at $676 \mathrm{~nm}\left(b_{p}(676)\right.$, as given in Figure 2c in [27]) to also be representative for $b_{p}(670)$.

Finally, the relationship between $a_{p}(670)$ and $b_{p}(670)$ had to be constrained, as particulate absorption and scattering in different waters are not 
independent, but are related through particle concentration. We have calculated the range of particulate scattering to absorption ratios from the singlescattering albedo range for particles at $670 \mathrm{~nm}$ $\left(\omega_{p}(670)\right)$ in Figure 3c in [27]:

$$
\frac{b_{p}(670)}{a_{p}(670)}=\left(\omega_{p}^{-1}-1\right)^{-1}
$$

We used this information to define a region in the $a_{p}-b_{p}$ space that respects all three conditions on $a_{p}(670), b_{p}(670)$, and $b_{p}(670) / a_{p}(670)$. To create a distribution of IOPs similar to the natural one, we have selected values of the particulate absorption and scattering at equal logarithmic intervals. We chose ten different values for $a_{p}(670)$, and, for each of them, ten different values for $b_{p}(670)$ so that the range for $b_{p}(670) / a_{p}(670)$ is respected. For the lowest value of the absorption coefficient, we discarded nine of the scattering values and kept only one, as the small variability in $b_{p}(670)$ for that case did not justify the computational effort in simulating all ten different cases. A separate MATLAB procedure calculating the IOP forcing values used to drive the optical simulations is available at http://misclab .umeoce.maine.edu/code/IOP_inversion_model/.

\section{Appendix B: Sensitivity Analyses}

\section{Petzold VSF}

To further test the sensitivity of our inversion algorithm to the VSF, we performed two simulations where particle scattering was described by the empirically determined Petzold average-particle phase function [16]. We ran the simulation for two IOP combinations, one for which the inversion algorithm performed very well on the data obtained from a Fournier-Forand model run $\left(a_{p}=\right.$ $0.005 \mathrm{~m}^{-1}, b_{p}=0.116 \mathrm{~m}^{-1}$ ) and one for which the inversion had the worst performance $\left(a_{p}=0.17 \mathrm{~m}^{-1}\right.$, $\left.b_{p}=6.398 \mathrm{~m}^{-1}\right)$. The results of these tests are shown in Table 6 .

To assess the significance of the obtained differences in the inversion performance on the Petzold and Fournier-Forand data sets, we used an ensemble of identical simulations using the Fournier-Forand scattering phase function with $B_{p}=0.02$ to estimate the relative errors in the IOP inversions introduced by Monte Carlo noise alone. We found that inversion

\begin{tabular}{|c|c|c|c|}
\hline Bp & Parameter & $\begin{array}{c}\text { Instrument } \\
\text { on Water } \\
\text { Surface }\end{array}$ & $\begin{array}{l}\text { Instrument } \\
\text { in Water }\end{array}$ \\
\hline \multirow{5}{*}{0.025} & $D$ & 0.0101 & 0.0100 \\
\hline & $\alpha\left(\mathrm{m}^{-1}\right)$ & 0.316 & 0.303 \\
\hline & $\varepsilon_{b b}$ & $-0.6 \%$ & $-4.9 \%$ \\
\hline & $\varepsilon_{a}$ & $27.5 \%$ & $23.3 \%$ \\
\hline & $D$ & 0.0060 & 0.0060 \\
\hline \multirow[t]{4}{*}{0.015} & $\alpha\left(\mathrm{m}^{-1}\right)$ & 0.192 & 0.185 \\
\hline & $\varepsilon_{b b}$ & $-1.6 \%$ & $-5.5 \%$ \\
\hline & $\varepsilon_{a}$ & $6.8 \%$ & $2.5 \%$ \\
\hline & $D$ & 0.0020 & 0.0020 \\
\hline \multirow[t]{3}{*}{0.005} & $\alpha\left(\mathrm{m}^{-1}\right)$ & 0.063 & 0.065 \\
\hline & $\varepsilon_{b b}$ & $-8.0 \%$ & $-5.9 \%$ \\
\hline & $\varepsilon_{a}$ & $-26.2 \%$ & $-25.1 \%$ \\
\hline
\end{tabular}

errors due to random noise are up to $7.6 \%$ for $b_{b}$ and up to $7 \%$ for $a$. Since, for both tests presented in Table $\underline{6}$, the differences in the inversion algorithm performance between the Petzold and the Fournier-Forand simulations are less than the Monte Carlo noise-introduced errors, we conclude that the IOP inversion algorithm performed equally well on simulations using the two different VSFs.

\section{Proximity to Water Surface}

To test for the sensitivity of the inversion algorithm to the vicinity of the instrument to the reflective water surface, we performed three more model runs in which we removed the water-air boundary (simulating the instrument underwater, infinitely far from the surface). The vicinity of the water surface should have the strongest effect on the instrument retrieval when the scattering is highest and the absorption is lowest (when more photons that reflect internally onto the water-air interface are likely to be scattered back into the detector). To assess the maximum error introduced by the presence of this boundary, we ran this model for the highest single-scattering albedo $(b / c)$ value used in our simulations for three different values of the backscattering ratio. Table 7 shows the comparison between the in-water versus on-water model runs for the two main signal descriptors $(D$ and $\alpha)$ and for the relative errors in retrieving $a$ and $b_{b}$ when the inversion algorithm based on Eqs. (13) and (14) was used on the modeled signals.

Table 6. Inversion Algorithm Performance on Simulated Detector Response: Comparison between Numerical Experiments Using Different Scattering Phase Functions ${ }^{a}$

\begin{tabular}{lccccc}
\hline Experiment & $b_{b, \text { real }}\left(\mathrm{m}^{-1}\right)$ & $b_{b, \text { inverted }}\left(\mathrm{m}^{-1}\right)$ & Relative Error $b_{b}(\%)$ & $a_{\text {real }}\left(\mathrm{m}^{-1}\right)$ & $a_{\text {inverted }}\left(\mathrm{m}^{-1}\right)$ \\
\hline P1 & $2.5 \times 10^{-3}$ & $2.6 \times 10^{-3}$ & -2.5 & 0.44 & 0.45 \\
FF1 & $2.7 \times 10^{-3}$ & $2.7 \times 10^{-3}$ & -0.8 & 0.44 & 0.45 \\
P2 & 0.117 & 0.128 & -8.8 & 0.6 & 0.84 \\
FF2 & 0.128 & 0.138 & -7.6 & 0.6 & 0.86 \\
\hline
\end{tabular}

${ }^{a} \mathrm{P} 1$ and P2 use the Petzold scattering phase function $\left(B_{p}=0.0183\right)$ and FF1 and FF2 use the Fournier-Forand phase function $\left(B_{p}=0.02\right)$ to simulate scattering by particles. $\mathrm{P} 1$ and FF $1, a_{p}=0.005 \mathrm{~m}^{-1}, b_{p}=0.116 \mathrm{~m}^{-1} ; \mathrm{P} 2$ and FF2, $a_{p}=0.17 \mathrm{~m}^{-1}, b_{p}=6.398 \mathrm{~m}^{-1}$. 
For $B_{p}=0.005$, the availability of an ensemble of identical simulations (instrument on the water surface) enabled us to assess the relative errors associated to the IOP inversions introduced by Monte Carlo noise. We found these errors to be up to $4.5 \%$ for the inversion of $b_{b}$ and up to 3\% for the inversion of $a$. Since the differences in the inversion algorithm performance between the on-water and in-water simulations are less than the Monte Carlo noiseintroduced errors, we conclude that the IOP inversion algorithm performed equally well on data from a simulated submerged instrument and data from an instrument simulated on the water surface.

We gratefully acknowledge Nick Record and the Gulf of Maine Research Institute for providing us with most of the computation time for the optical simulations and Thomas Leeuw for assistance with the laboratory experiment photography. We also thank two anonymous reviewers and Chuanmin $\mathrm{Hu}$ for comments that improved the quality of this paper. Financial support for this project was offered by the University of Maine (through a Provost Fellowship to A. Gainusa Bogdan) and the Environmental Optics program of the Office of Naval Research (ONR) (support for E. Boss through grant N0001404-1-0235).

\section{References and Notes}

1. C. Giardino, V. E. Brando, A. G. Dekker, N. Strombeck, and G. Candiani, "Assessment of water quality in lake Garda (Italy) using Hyperion," Remote Sens. Environ. 109, 183-195 (2007).

2. T. Dickey, M. Lewis, and G. Chang, "Optical oceanography: recent advances and future directions using global remote sensing and in situ observations," Rev. Geophys. 44, RG1001 (2006).

3. C. S. Roesler and E. Boss, "In situ measurement of the inherent optical properties (IOPs) and potential for harmful algal bloom detection and coastal ecosystem observations," in RealTime Coastal Observing Systems for Ecosystem Dynamics and Harmful Algal Bloom (UNESCO, 2008), pp. 153-206.

4. Z. Lee, "Remote sensing of inherent optical properties: fundamentals, tests of algorithms, and applications," Tech. Rep. 5 (International Ocean Colour Coordinating Group, 2006).

5. R. F. Cahalan, M. McGill, J. Kolasinski, T. Várnai, and K. Yetzer, "THOR-Cloud thickness from offbeam lidar returns," J. Atmos. Ocean. Technol. 22, 605-627 (2005).

6. K. Suzuki, M. Kishino, K. Sasaoka, S. Saitoh, and T. Saino, "Chlorophyll-specific absorption coefficients and pigments of phytoplankton off Sanriku, northwestern north Pacific," J. Oceanogr. 54, 517-526 (1998).

7. J. Downing, "An optical instrument for monitoring suspended particulates in ocean and laboratory," in Proceedings OCEANS '83 (IEEE, 1983), pp. 199-202.

8. Absorption measurements have been performed using ac-9+ spectrophotometers mounted on very large AUVs [28,29]. However, because of their size (requiring large vessels for deployment and implying short mission durations), these platforms are not applicable to long-term routine IOP monitoring.

9. M. S. Twardowski, E. Boss, J. B. Macdonald, W. S. Pegau, A. H. Barnard, and J. R. V. Zaneveld, "A model for estimating bulk refractive index from the optical backscattering ratio and the implications for understanding particle composition in case I and case II waters," J. Geophys. Res. 106, 14129-14142 (2001).

10. E. Boss, W. S. Pegau, M. Lee, M. Twardowski, E. Shybanov, G. Korotaev, and F. Barantange, "Particulate backscattering ratio at LEO 15 and its use to study particlecomposition and distribution," J. Geophys. Res. 109, C01014 (2004).

11. J. M. Sullivan, M. S. Twardowski, P. L. Donaghay, and S. A. Freeman, "Use of optical scattering to discriminate particle types in coastal waters," Appl. Opt. 44, 1667-1680 (2005).

12. H. Loisel, X. Mériaux, J.-F. Berthon, and A. Poteau, "Investigation of the optical backscattering to scattering ratio of marine particles in relation with their biogeochemical composition in the eastern English Channel and southern North Sea," Limnol. Oceanogr. 52, 739-752 (2007).

13. A. L. Whitmire, E. Boss, T. J. Cowles, and W. S. Pegau, "Spectral variability of the particulate backscattering ratio," Opt. Express 15, 7019-7031 (2007).

14. G. R. Fournier and M. Jonasz, "Computer-based underwater imaging analysis," in Conference on Airborne and In-Water Underwater Imaging (SPIE, 1999), Vol. 3761, pp. $62-70$.

15. C. D. Mobley, L. K. Sundman, and E. Boss, "Phase function effects on oceanic light fields," Appl. Opt. 41, 1035-1050 (2002).

16. C. D. Mobley, Light and Water: Radiative Transfer in Natural Waters, 1st ed. (Academic, 1994).

17. R. A. Leathers, T. V. Downes, C. O. Davis, and C. D. Mobley, "Monte Carlo radiative transfer simulations for ocean optics: a practical guide," Memorandum report A426624 (Naval Research Laboratory, 2004).

18. R. Maffione and D. R. Dana, "Instruments and methods for measuring the backward-scattering coefficient of ocean waters," Appl. Opt. 36, 6057-6067 (1997).

19. E. Boss and W. S. Pegau, "Relationship of light scattering at an angle in the backward direction to the backscattering coefficient," Appl. Opt. 40, 5503-5507 (2001).

20. E. Leymarie, D. Doxaran, and M. Babin, "Uncertainties associated to measurements of inherent optical properties in natural waters," Appl. Opt. 49, 5415-5436 (2010).

21. J. R. V. Zaneveld, J. C. Kitchen, A. Bricaud, and C. C. Moore, "Analysis of in-situ spectral absorption meter data," Proc. SPIE 1750, 187-200 (1992).

22. D. McKee, M. Chami, I. Brown, V. S. Calzado, D. Doxaran, and A. Cunningham, "Role of measurement uncertainties in observed variability in the spectral backscattering ratio: a case study in mineral-rich coastal waters," Appl. Opt. 48, 46634675 (2009).

23. T. K. Westberry, G. Dall'Olmo, E. Boss, M. J. Behrenfeld, and T. Moutin, "Coherence of particulate beam attenuation and backscattering coefficients in diverse open ocean environments," Opt. Express 18, 15419-15425 (2010).

24. D. L. Rudnick and M. J. Perry, "ALPS: Autonomous and Lagrangian Platforms and Sensors," Workshop report, www .geo-prose.com/ALPS (Geo Prose, 2003).

25. T. D. Dickey, E. C. Itsweire, M. A. Moline, and M. J. Perry, "Introduction to the limnology and oceanography: special issue on autonomous and Lagrangian platforms and sensors (ALPS)," Limnol. Oceanogr. 53, 2057-2061 (2008).

26. M. Babin, D. Stramski, G. M. Ferrari, H. Claustre, A. Bricaud, G. Obolensky, and N. Hoepffner, "Variations in the light absorption coefficients of phytoplankton, nonalgal particles, and dissolved organic matter in coastal waters around Europe," J. Geophys. Res. 108, 3211 (2003). 
27. M. Babin, A. Morel, V. Fournier-Sicre, F. Fell, and D. Stramski, "Light scattering properties of marine particles in coastal and open ocean waters as related to the particle mass concentration," Limnol. Oceanogr. 48, 843-859 (2003).

28. A. Cunningham, D. McKee, S. Craig, G. Tarran, and C. Widdicombe, "Fine-scale variability in phytoplankton community structure and inherent optical properties measured from an autonomous underwater vehicle," J. Mar. Syst. 43, 51-59 (2003).
29. W. Wijesekera, W. S. Pegau, and T. J. Boyd, "Effect of surface waves on the irradiance distribution in the upper ocean," Opt. Express 13, 9257-9264 (2005).

30. A. H. Barnard, W. S. Pegau, and J. R. V. Zaneveld, "Global relationships of the inherent optical properties of the oceans," J. Geophys. Res. 103, 24955-24968 (1998).

31. N. J. Jerlov, "Marine optics," Elsevier Oceanogr. Ser. 14, i-xiii, 1-231 (1976). 